Código JEL: M310

\title{
El análisis de diseño de sitio web de las mayores empresas siderúrgicas chinas a través de la lógica borrosa
}

\author{
Xxx DUOJIEZHAXI \\ Universidad Complutense de Madrid \\ xxduojie@ucm.es \\ Javier DE ESTEBAN CURIEL \\ Universidad Rey Juan Carlos \\ javier.deesteban@urjc.es \\ Arta ANTONOVICA \\ Universidad Rey Juan Carlos \\ arta.antonovica@urjc.es
}

Recibido: 30-10-2016

Aceptado: 20-11-2016

\begin{abstract}
RESUMEN
El sitio web de la empresa es un buen mecanismo para facilitar una comunicación eficaz entre proveedores y clientes, y es una de las técnicas más eficientes para articular las relaciones comunicativas y comerciales. En esta investigación, se ha utilizado la lógica difusa para la analítica web. El enfoque se ha basado en las 7C de diseño del sitio web creado por Jeffrey y Jaworski (2001). Se observa que la lógica borrosa permite alcanzar soluciones que se aproximan más a nuestra realidad en el caso de la analítica web. Así, este trabajo es un estudio comparativo, y su propósito es contrastar el rendimiento de diseño del sitio web de las 25 empresas fabricantes y las 25 empresas intermediarias del sector de acero de China con los mejores números de facturación de ventas de acero en el año 2014.
\end{abstract}

Palabras clave: Lógica Borrosa, Lógica Difusa, Diseño de Sitio Web, Analítica Web y Estudio Comparativo. 


\title{
Analysis of Website Design of China's Largest Steel Companies through Fuzzy Logic
}

\begin{abstract}
Corporate websites are a good mechanism for facilitating effective communication between suppliers and customers, and are one of the most effective techniques to facilitate communication and trading relations. In this research, the fuzzy logic has been applied for web analytics, focusing on the 7Cs of web design created by Jeffrey and Jaworski (2001). We observed that fuzzy logic allows reaching solutions that are closer to our reality in the case of web analytics. This work is a comparative study, and its purpose is to compare the performance of web design of the top 25 manufacturing companies and the top 25 trading companies of China's steel sector, with the best ranges of steel sales turnover in 2014.
\end{abstract}

Keywords: Fuzzy Logic, Fuzzy Logic, Website Design, Web Analytics and Comparative Study.

Sumario: 1. Introducción 2. Marco Teórico. 3. Metodología. 4. Análisis e Interpretación de los Resultados con Análisis Fuzzy. 5. Conclusiones. 6. Bibliografía.

\section{INTRODUCCIÓN}

La influencia de sitio web en la sociedad contemporánea es muy grande, ya que los vendedores continuamente utilizan el sitio web para informar, entretener, controlar y formar a los clientes, a través de su constante evolución en distintos formatos, como la web estática y la dinámica. Hacerlo bien y saberlo bien es fundamental para el éxito de la empresa en la era nueva. Al contrario si no toma en cuenta el protagonismo del sitio web en la sociedad moderna, la empresa puede claudicar los clientes nuevos en los primeros años de haberse constituido, hasta perder muchos clientes ya existentes.

Como el acero es un material que tiene mucha demanda y competencia en el mercado, el desafío que supone el sitio web en este sector sirve de impulso para mejorar y desarrollar la forma de negocio especialmente para las empresas que todavía no son conscientes de la importancia de TIC. Lo que queremos decir que es el buen aprovechamiento de sitio web permite que muchas empresas que lo desean puedan negociar.

La presente investigación se centra en el uso de las $7 \mathrm{C}$ de la interfaz para el consumidor de sitio web creado por Jeffrey y Jaworski (2001). El objetivo principal de esta investigación es utilizar la lógica difusa en el análisis de las $7 \mathrm{C}$ de sitio web de las 25 mejores empresas fabricantes y las 25 mejores empresas 
intermediarias del sector de acero de China, que tienen los mejores rangos de la facturación de ventas en el año 2014. Para finalizar, se realiza una comparación de resultados entre el rendimiento de las $7 \mathrm{C}$ de diseño de sitio web de los dos participantes mencionados anteriormente.

\section{MARCO TEÓRICO}

El sitio web es un elemento fundamental en la estrategia de negocio en Internet (Tejedor, R. J., 2008). La web social permite la interacción de organizaciones y clientes, y propicia entornos comunes que facilitan la adopción de nuevas alternativas para presentar a los clientes (Ribes, 2007; citado en Gusmán, 2013). Una de las formas mas usadas para evaluar y desarrollar un análisis crítico del diseño general de un sitio web es el modelo de las $7 \mathrm{C}$ de Jeffrey Rayport y Bernard Jaworski (2001), enunciado en el libro E-Commerce. Básicamente, evalúa por separado los siete elementos que considera relevantes, que son: el contexto, el contenido, la comunidad, la conversión, la comunicación, la conexión, y el comercio. Aunque, Andrew Yang y et al, (2008; Citado por Sabouri y Jalali, 2009:1693) denominaron 8C el "Cambio" y completaron el modelo de las 7 C de Jeffrey y Jaworski (2001) en 2008, en nuestra investigación, vamos a seguir el modelo de Jeffrey y Jaworski (2001).

Las medidas seleccionadas para la presente investigación están basadas en el modelo de la evaluación de métricas de rendimiento de 7C de diseño de sitio web de Jeffrey y Jaworski (2001) y Ranawaka (2008). Sin embargo, hemos desarrollado el concepto de cada una de estas 10 medidas seleccionadas específicamente para tener una evaluación objetiva y satisfactoria en nuestro estudio. Mientras que muchas pautas del diseño de sitio web que recoge la literatura parecen tener validez, en algún momento se carece de especificidad y mensurabilidad. A continuación, se presenta una descripción detallada de cada medida para nuestro estudio, junto con su fuente de información:

- Medida 1: Función y estética. Función. La extensa cantidad de información debe presentarse al cliente de modo coherente para que pase de un punto de interés a otro dentro del sitio web. Un sitio bien diseñado organiza toda la información en series de páginas y ofrece a los clientes medios para navegar de una página a otra. Estética (color/gráficos/animación). La naturaleza estética del sitio se crea a través de características visuales como colores, gráficos, fotografías, opciones de tipo de letra y otros rasgos de carácter visual. (Jeffrey y Jaworski; 2001:140).

- Medida 2: Mezcla de multimedia y mezcla de ofertas. Mezcla de multimedia se refiere a las opciones de medios que incluyen texto, audio, imagen, vídeo y gráficos. Mezcla de ofertas se refiere al contenido, donde se puede incluir 
productos, información o servicios. En muchas ocasiones los sitios incluyen una combinación de estos tres elementos (Jeffrey y Jaworski; 2001).

- Medida 3: Mezcla de atracción. La mezcla de atracción se refiere a los mensajes de promoción y comunicación que proyecta la compañía. Por naturaleza, uno esperaría que la mezcla de atracción esté muy vinculada con la propuesta de valor. La bibliografía académica identifica dos atractivos generales: cognoscitivo y emocional. (Jeffrey y Jaworski; 2001).

- Medida 4: Evidencia de actualización periódica. El objetivo de incluir este indicador obedece a poder determinar un criterio de temporalidad a través del cual el usuario pueda comprobar el nivel de actualización de los contenidos de la web (González, n.d.). Hay que señalar que los resultados de la evidencia de actualización periódica, obtenidos desde el Internet Archive (2015), se basa en la exclusiva naturaleza de la actualización de sitio web de la empresa, y está disponible en https://web.archive.org/web.

- Medida 5: Comunicación interactiva entre los usuarios. Los usuarios o clientes pueden intercambiar respuestas de manera directa y continua como una forma de comunicación interactiva. Los sitios proporcionan facilidades para sustentar una comunicación interactiva de usuario a usuario en tiempo real o casi real como conversaciones electrónicas en varias formas, tales como: plática electrónica, mensajes o pláticas electrónicas instantáneas, tableros de mensajes, correo electrónico de miembro a miembro (Jeffrey \& Jaworski; 2001).

- Medida 6: Capacidad de adaptación y personalización. Un sitio puede usar un motor de recomendación para adaptarse de forma automática al comportamiento de cada usuario y variar la mezcla de ofertas de productos, servicios e información del sitio (Jeffrey \& Jaworski (2001). Una vez que el usuario aporta sus preferencias, el sitio usa un registro o cookie para concordar tales preferencias cuando el usuario regresa. El sitio se configura de acuerdo con estas preferencias. (Jeffrey \& Jaworski (2001).

- Medida 7: La comunicación entre el sitio y el usuario. La comunicación entre el sitio y el usuario se refiere la comunicación interactiva, que es la comunicación bilateral entre la organización y un usuario (Jeffrey y Jaworski (2001).

- Medida 8: Disponibilidad de enlaces de sitio web de la empresa. Los enlaces son el principal medio de navegación en la web (Johnson, 2003). En la presente métrica, se mide la disponibilidad de enlaces externos del sitio web de la empresa a través de PageRank o el rango de la página. Hay que señalar que los resultados de esta medida están obtenidos desde PageRank, y está disponible en http://checkpagerank.net. En este estudio, suponemos que el número máximo de páginas externas que pueda llegar un sitio web de una empresa mundial, como sucede con el caso que nos ocupa, es de cien mil, y los 
datos estadísticos de este apartado son divididos por $10^{6}$ para ajustar entre los valores numéricos 0 y 1 .

- Medida 9: El rango de la página. El PageRank es un valor numérico (contabilizado de 0 al 10) que representa la relevancia de una página web. Google contabiliza cuando una web enlaza a otra web, cuantas más webs te enlacen, el buscador Google dará mas importancia a tu web y por lo tanto más puntuación. (Calcularpagerank.com, 2009). El PageRank se basa en la naturaleza exclusivamente democrática de la web y usa su extensa estructura de vínculos como indicador del valor de una página individual. Carreras Lario, 2012:40). Hay que señalar que los resultados de PageRank están obtenidos desde la página web de PageRank (2015), que está disponible en http://checkpagerank.net. En este estudio, suponemos que el valor numérico más alto 10 de PageRank como 1 y el número 1 de Pagerank como 0.1 para ajustar entre los valores numéricos 0 y 1 .

- Medida 10: Capacidad de las transacciones bancarias que realizan en línea. Los sitios pueden tener la capacidad de recibir aprobación de crédito instantánea para compras con tarjetas de crédito a través de vínculos electrónicos con las compañías correspondiente (Jeffrey \& Jaworski, 2001). De hecho, en este apartado, se observa si hay la posibilidad de hacer las transacciones bancarias en línea en el sitio web de la empresa o no. En caso de que exista, queda constancia de si la compra con tarjetas de crédito está activado o desactivado, y si tiene un link que nos dirige hacia sus transacciones bancarias que realizan en línea o no, y se da un 0.5 para los sitios que nos dirigen hacia su plataforma electrónica.

\section{METODOLOGÍA}

La técnica de investigación empleada para la evaluación de las $7 \mathrm{C}$ de diseño de sitio web de las empresas siderúrgicas chinas es la lógica borrosa. La lógica borrosa nace en 1965 de la mano del profesor Lofti A. Zadeh, básicamente con el fin de enfrentarse a algunos de los problemas que presenta la lógica binaria clásica. La lógica binaria es a veces inadecuada para la descripción del razonamiento humano, puesto que todo se define como 0 (Falso) o 1 (Verdadero) (Martín Delbrío 2001; citado en León de Mora, 2009: 68).

La lógica borrosa usa el intervalo completo comprendido entre 0 y 1 para caracterizar dicho razonamiento, por lo que se permiten los estados intermedios (Jantzen 98; citado en Ropero, 2009: 68). En la toma de decisión multicriterio, comúnmente, se presentan criterios subjetivos no cuantitativos, esto debido en gran medida a que no siempre es fácil realizar una adecuada ponderación de criterios con los métodos cuantitativos. Por esta razón, se utilizan técnicas de lógica difusa 
que permiten transformar variables lingüísticas (Liu \& Wang, 2006; citado en Vera, 2014).

La lógica fuzzy permite el traslado de medidas a variables lingüísticas, de tal manera que permite la formación de conjunto de datos a diferentes escalas. Para el caso de las decisiones a nivel agrícola, fuzzy permite crear variables lingüísticas que normalmente otros métodos de evaluación multicriterio no permiten, creando conjuntos en base a las reglas de evaluación planteadas (Vera, 2014).

\title{
Conjuntos borrosos
}

Según Zadeh (1965:339), el concepto de los conjuntos borrosos como: un conjunto borroso $A$ en $X$ es caracterizado por una función de pertenencia (membership function) $f \mathrm{~A}(x)$ que asocia cada punto en $X$ un número real en el intervalo [0,1], en el que el valor de $f \mathrm{~A}(x)$ en $x$ representando el grado de pertenencia de $x$ en $A$; cuanto más se aproxima el valor de $f \mathrm{~A}(x)$ a la unidad, mayor es el grado de pertenencia de $x$ en $A$.

Zadeh (1965) identifica cuantificadores del lenguaje "mucho", "muy" y "un poco", interpretando además las operaciones de unión, intersección, diferencia, negación o complemento, y otras operaciones sobre conjuntos. Además Zadeh (1965) desarrolla algunos conceptos para este tipo de conjuntos mediante una generalización de los ya existentes para ordinarios, tales como: igualdad, complemento, inclusión, unión, intersección, disyunción y conjunto vacío. (Vera, 2014).

\section{Variables de entrada}

La lógica difusa necesita la creación de conjuntos sin límites totalmente definidos, con esto se entiende que puede existir intermedios entre la pertenencia y no pertenencia a un conjunto. Para poder entender claramente cómo se conceptualiza estas funciones de pertenencia, se utilizan expresiones lingüísticas de las variables que en su momento Zadeh (1975) lo conceptualizó así:

\begin{abstract}
"por una variable lingüística se quiere decir que se trata de una variable cuyos valores son palabras u oraciones en un lenguaje natural o artificial. La motivación para el uso de palabras o de oraciones en lugar de números es que las caracterizaciones lingüísticas son, en general, menos precisas que los valores numéricos"
\end{abstract}

Según Zadeh (1994), un concepto que juega un papel básico en las aplicaciones de la lógica difusa es la del uso de una variable lingüística. Existe, sin embargo, un aspecto básico del concepto de una variable lingüística que es digno de resaltar su utilidad. Un ejemplo en concreto, es considerar una variable lingüística "joven"; mientras que en los conjuntos ordinarios se deben definir edades concretas, en el 
caso de lógica difusa una variable lingüística puede representar la opción joven con varios valores posibles (Vera, 2014).

\section{Funciones de pertenencia}

La función de pertenencia se define de tal modo que la imagen de los elementos que pertenecen con seguridad al conjunto es 1 y la de los que con seguridad no pertenecen es 0 ; en cualquier otro caso la imagen será un valor de $[0,1]$ que estará tanto más próxima a uno de los extremos cuanto más nos aproximemos a esa certeza, pero sin llegar a confiar totalmente en ella. El caso de clara duda y desconfianza corresponde a la imagen 0,5 (centro del intervalo) (Gómez, 2010).

Las tres operaciones básicas entre conjuntos concretos son (Vera, 2014):

- Unión: el resultado de efectuar la operación de Unión entre dos conjuntos difusos $A$ y $B$ definidos sobre el mismo Universo, y con funciones de pertenencia $u \mathrm{~A}(x)$ y $u \mathrm{~B}(x)$ respectivamente, es un nuevo conjunto difuso $A \mathrm{UB}$ definido sobre el mismo universo, y con función de pertenencia $u$ $\operatorname{AUB}(x)$ :

$$
u \mathrm{AU}(x)=u \mathrm{~A}(x)(+) u \mathrm{~B}(x):
$$

- Complemento: el resultado de efectuar la operación de Complemento sobre un conjunto difuso $A$ definido sobre un Universo, y con función de pertenencia $u \mathrm{~A}(x)$ es un nuevo conjunto difuso $A^{\prime}$ definido sobre el mismo universo, y con función de pertenencia $u A^{\prime}(x)$ :

$$
u \mathrm{~A}^{\prime}(x)=1-u \mathrm{~A}(x)
$$

- Intersección: el resultado de efectuar la operación de Intersección entre dos conjuntos difusos $A$ y $B$ definidos sobre el mismo Universo, y con funciones de pertenencia $u \mathrm{~A}(x)$ y $u \mathrm{~B}(x)$ respectivamente, es un nuevo conjunto difuso $A \cap B$ definido sobre el mismo universo, y con función de pertenencia $u \mathrm{~A} \cap B(x)$ :

$$
u \mathrm{~A} \cap(x)=u \mathrm{~A}(x)(*) u \mathrm{~B}(x)
$$

\section{Reglas de evaluación de la Inferencia borrosa}

Como en la lógica clásica, la lógica difusa se ocupa del razonamiento formal con proposiciones, pero a diferencia de esta, los valores de las proposiciones pueden tener valores intermedios entre verdadero y falso. El concepto de variable lingüística fue un escalón al concepto de reglas difusas SI-ENTONCES. Estas son una base para la lógica difusa que a menudo es utilizada en aplicaciones prácticas (Zadeh, 1975, 1996; Rutkowska, 2002).

Wang (1997; citado en Vera, 2014:99) señala que el corazón de un sistema difuso es generar una base de conocimiento que consiste en la llamada regla difusa IF-THEN. En resumen, el punto de partida de la construcción de un sistema difuso es la obtención de una colección de reglas borrosas SI - ENTONCES de expertos 
humanos. El siguiente paso es combinar estas reglas en un único sistema. Diferentes sistemas difusos utilizan diferentes principios para esta combinación.

\section{Desborrosificación (Defuzzification)}

Para expresar un número en palabras, necesitamos una manera de traducir los valores numéricos de entrada en un conjunto borroso de descriptores lingüísticos: este es el llamado proceso de borrosificación (Pant, 2004; citado en León de Mora, 2009: 73).

La desborrosificación es, como su nombre indica, el proceso opuesto a la borrosificación. En el método de implicación (reglas SI... ENTONCES), cada regla es aplicada al número dado por el antecedente, construyéndose un conjunto borroso V para el consecuente. Este conjunto V es la salida de un dispositivo de inferencia borroso y, generalmente, suele ser convertido en un valor no borroso y $\epsilon$ V (León de Mora, 2009).

El método de desborrosificación que hemos utilizado en esta investigación es Centro de Gravedad. Con este método se tiene en cuenta información relacionada con la función de pertenencia $\mu \mathrm{B}$. Se toma la media de todos los pesos (Jang et al. 1997).

\section{ANÁLISIS E INTERPRETACIÓN DE RESULTADOS DE LA LÓGICA BORROSA}

Según los datos estadísticos de GLinfo.com [钢联资讯] (2014), Docin.com [豆丁网] (2014) y Mysteel.com (2014) y ASKCI Consulting [中商情报网], (2014), hemos elegidos sitios web de las 25 mejores empresas fabricantes del sector de acero de China y las 25 mejores empresas intermediarias del dicho sector del mismo país (véase en la Tabla 1), que tienen los mejores rangos de la facturación de ventas de acero en el año 2014. En total tenemos una muestra de 50 empresas siderúrgicas chinas, compuesto por dos categorías según la estructura de la empresa, por ejemplo, las empresas fabricantes de sector siderúrgico chino y las empresas intermediarias del dicho sector de China.

En nuestra investigación, se ha utilizado el modelo de las $7 \mathrm{C}$ y se ha desarrollado una lista de (10) medidas (c1.1, c1.2, ..., c7), con el fin de comparar los elementos del diseño de los sitios web y clasificar los sitios web de las empresas siderúrgicas chinas. El modelo propuesto tiene en cuenta veinticinco (25) empresas fabricantes chinas del sector de acero (F1, F2, ..., F25), como se muestra en la tabla 1; también tiene en cuenta otras veinticinco (25) empresas intermediarias (M1, M2, ..., M25), como se muestra en la misma tabla, para la evaluación del desempeño de las 7C de las empresas siderúrgicas chinas. Los 
modelos utilizaron el enfoque de la lógica difusa con el fin de proporcionar una solución razonable para el evaluador o el tomador de decisiones, para llevar a cabo una evaluación exhaustiva tanto de las 25 mejores empresas fabricantes como de las 25 mejores empresas intermediarias del sector de acero de China. También tiene por misión establecer un método más significativo de la evaluación de interfaz de usuario de las empresas siderúrgicas chinas.

Tabla 1. Las empresas participantes de nuestro estudio

\begin{tabular}{|c|c|c|c|}
\hline $\begin{array}{l}\text { Representación } \\
\text { de fabricantes }\end{array}$ & $\begin{array}{l}\text { Nombre de la empresa } \\
\text { fabricante }\end{array}$ & $\begin{array}{l}\text { Representación } \\
\text { de intermediarios }\end{array}$ & $\begin{array}{l}\text { Nombre de la empresa } \\
\text { intermediaria }\end{array}$ \\
\hline F1. & $\begin{array}{l}\text { Shanghai Baosteel Group } \\
\text { Corporation }\end{array}$ & M 1. & $\begin{array}{l}\text { China Railway Materials } \\
\text { Company Limited }\end{array}$ \\
\hline$\overline{\mathrm{F} 2 .}$. & Hebei Iron \& Steel Group & M 2. & $\begin{array}{l}\text { Minmetals Development } \\
\text { Co., Limited }\end{array}$ \\
\hline F3. & Shagang Group & M 3. & $\begin{array}{ll}\text { Zhejiang } & \text { Materials } \\
\text { Industry Group } & \end{array}$ \\
\hline F4. & Wuhan Iron and Steel Group & M 4. & Hangzhou Ciec Group \\
\hline F5. & Bohai steel group & M 5. & Sinosteel Corporation \\
\hline F6. & Shougang group & M 6. & Huanan Group \\
\hline F7. & $\begin{array}{l}\text { Xinxing Cathay International } \\
\text { Group }\end{array}$ & M 7. & Xiangyu Group \\
\hline F8. & Ansteel group & M 8. & $\begin{array}{l}\text { Southern Building } \\
\text { Materials Co., Ltd. }\end{array}$ \\
\hline F9. & Taiyuan Iron \& Steel Group & M 9. & Junhe Group \\
\hline F10. & Jiuquan Iron \& Steel Group & M 10. & $\begin{array}{l}\text { Shanghai Miaoshi Group } \\
\text { Co.,Ltd }\end{array}$ \\
\hline F11. & Shandong Iron \& Steel Group & M 11. & $\begin{array}{l}\text { Shanghai Baiying Iron \& } \\
\text { Steel Group }\end{array}$ \\
\hline F12. & Benxi Iron \& Steel Group & M 12. & $\begin{array}{l}\text { Xiamen } \quad C \& D \quad \text { Material } \\
\text { Co., Ltd }\end{array}$ \\
\hline F13. & Zenith Steel Group & M 13. & Huaye Iron \& Steel Group \\
\hline F14. & $\begin{array}{l}\text { Hangzhou Iron \& } \\
\text { Group }\end{array}$ & M 14. & $\begin{array}{l}\text { Shanghai Minlurun Trade } \\
\text { Co.,Ltd }\end{array}$ \\
\hline F15. & Valin group & M 15. & $\begin{array}{l}\text { Chang Jiang Steel Pipe } \\
\text { Co., Ltd }\end{array}$ \\
\hline F16. & Magang Group & M 16. & $\begin{array}{l}\text { Shanghai Ningjin Iron \& } \\
\text { Steel Co., Ltd }\end{array}$ \\
\hline F17. & Jianlong Group & M 17. & $\begin{array}{l}\text { Shanghai Jushen Metal } \\
\text { Industry Co., Ltd }\end{array}$ \\
\hline
\end{tabular}




\begin{tabular}{|l|l|l|l|}
\hline F18. & Sino Giant Group & M 18. & $\begin{array}{l}\text { Shanghai Shunye Steel } \\
\text { Co., Ltd }\end{array}$ \\
\hline F19. & Nanjing Iron \& Steel Group & M 19. & $\begin{array}{l}\text { Shanghai Ronghui Wuzi } \\
\text { Co., Ltd }\end{array}$ \\
\hline F20. & $\begin{array}{l}\text { Jiangsu Xicheng Sanlian } \\
\text { Holding Group }\end{array}$ & M 20. & Golden Jumping Group \\
\hline F21. & Fosun Group & M 21. & $\begin{array}{l}\text { Shanghai Qiangguan Steel } \\
\text { Co., Ltd }\end{array}$ \\
\hline F22. & Jinxi Group & M 22. & Qixin Group of Hebei \\
\hline F23. & Baogang Group & M 23. & Zhanzhi Group \\
\hline F24. & Hebei JIngye Group & M 24. & $\begin{array}{l}\text { Beijing Congtie } \\
\text { Construct Materials Ltd }\end{array}$ \\
\hline F25. & Tranvic Group & M 25. & $\begin{array}{l}\text { Shanghai } \\
\text { International Co., Ltd }\end{array}$ \\
\hline
\end{tabular}

Fuente: Elaboración propia.

\section{Fusificación}

Para analizar el grado de pertenencia, a continuación formamos los siguientes cinto conjuntos difusos en la tabla 5 para describir el desempeño de las $7 \mathrm{C}$ de los sitios web de las empresas siderúrgicas chinas (Véase en la tabla 1).

Para calcular el grado de pertenencia (membership function), es necesario realizar las entradas fuzzy, determinar las funciones de pertenencia de cada variable y las etiquetas de cada una de ellas. Las variables de entrada seleccionadas fueron los criterios considerados para la evaluación de las 7C de diseño efectivo de sitios web de las empresas siderúrgicas chinas.

A continuación, se presentan las 10 medidas, que son 10 variables de entrada para la evaluación del rendimiento de las 7C de diseño de sitios web de las empresas siderúrgicas chinas (Véase en la tabla 2):

Tabla 2. Medidas de rendimiento de $7 \mathrm{C}$ de diseño de sitio web de las empresas siderúrgicas chinas

\begin{tabular}{|c|c|c|}
\hline $\begin{array}{c}\text { Componentes de la interfaz de } \\
\text { usuario }\end{array}$ & Subcomponentes & Representación \\
\hline Contexto & Función y estética. & $\mathrm{C} 1$ \\
\hline \multirow{2}{*}{ Contenido } & Mezcla de multimedia y mezcla de ofertas. & $\mathrm{C} 2.1$ \\
\cline { 2 - 3 } & Mezcla de atracción. & $\mathrm{C} 2.2$ \\
\cline { 2 - 3 } & Evidencia de actualización periódica. & $\mathrm{C} 2.3$ \\
\hline Comunidad & Comunicación interactiva entre los usuarios. & $\mathrm{C} 3$ \\
\hline Conversión & Capacidad de adaptación y personalización. & $\mathrm{C} 4$ \\
\hline Comunicación & La comunicación entre el sitio y el usuario. & $\mathrm{C} 5$ \\
\hline
\end{tabular}




\begin{tabular}{|c|c|c|}
\hline \multirow{2}{*}{ Conexión } & Disponibilidad de enlaces externos a otros sitios. & C 6.1 \\
\cline { 2 - 3 } & El rango de la página (PageRank) & C 6.2 \\
\hline Comercio & $\begin{array}{c}\text { Capacidad de las transacciones bancarias que } \\
\text { realizan en línea. }\end{array}$ & C 7 \\
\hline
\end{tabular}

Fuente: Elaboración propia.

A fin de poder obtener la valoración de los mejores tratamientos mediante la metodología fuzzy, se crearon las implicaciones entre los conjuntos difusos de entrada y el conjunto difuso de salida en este trabajo (Véase en la tabla 3). La tabla 3 ilustra las 36 reglas de evaluación donde se estructura metodológicamente lo ha realizado en el trabajo, a través de 3 variables de entrada, tales como la unión, la intersección y la media de las 10 variables mencionadas anteriormente, Para el caso de la variable de salida, se consideró el nivel de rendimiento del sitio web de la empresa.

\section{Las etiquetas para cada variable se describen a continuación:}

Variables de entrada: la función de pertenencia "la unión" de 10 variables de 7C de sitios web, la función de pertenencia, "la intersección" de 10 variables de 7C de sitios web, y el promedio de los resultados de las 10 variables de 7C.

- Etiquetas de la función de pertenencia, "la unión = $u \mathrm{AU}(x)=u \mathrm{~A}(x)(+) u \mathrm{~B}(x)$ " de las 10 variables: "Muy pobre", "Inadecuado", "Mediano", "Bien" y "Excelente".

- Etiquetas de la función de pertenencia "la intersección= $u \mathrm{~A} \cap B(x)=u \mathrm{~A} \quad(x)(*) u \mathrm{~B}$ " de las 10 variables: "Muy pobre", "Inadecuado", "Mediano", "Bien" y "Excelente".

- Etiquetas de promedio de los resultados de las 10 variables: "Muy pobre", "Inadecuado", "Mediano", "Bien" y "Excelente".

Variable de salida: evaluación de rendimiento del sitio web de la empresa.

- Etiquetas de evaluación de rendimiento del sitio web: "Muy pobre", "Inadecuado", "Mediano", "Bien" y "Excelente". 
Tabla 3. Reglas de evaluación de Fuzzy para el rendimiento del diseño de sitio web de las empresas siderúrgicas chinas

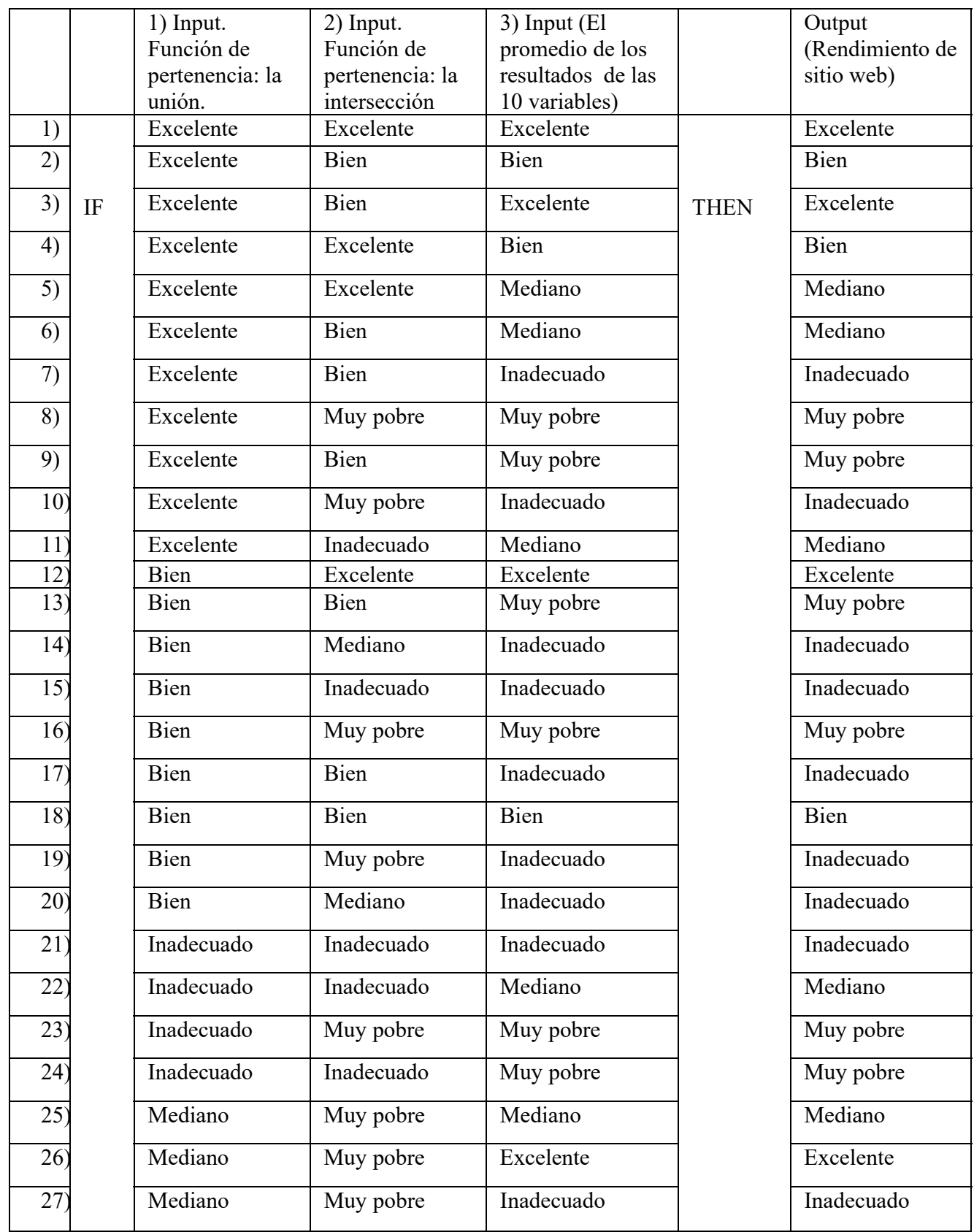




\begin{tabular}{|c|c|c|c|c|}
\hline 28) & Muy pobre & Mediano & Mediano & Mediano \\
\hline 29) & Muy pobre & Muy pobre & Muy pobre & Muy pobre \\
\hline 30) & Muy pobre & Inadecuado & Inadecuado & Inadecuado \\
\hline 31) & Muy pobre & Inadecuado & Muy pobre & Muy pobre \\
\hline 32) & Muy pobre & Inadecuado & Mediano & Mediano \\
\hline 33) & Muy pobre & Mediano & Inadecuado & Mediano \\
\hline 34) & Muy pobre & Inadecuado & Muy pobre & Muy pobre \\
\hline 35$)$ & Muy pobre & Mediano & Muy pobre & Muy pobre \\
\hline 36) & Muy pobre & Mediano & Inadecuado & Mediano \\
\hline
\end{tabular}

Fuente: Elaboración propia.

\section{Defusificación}

En nuestro estudio, se utilizó para la defusificación el método de "Centro de Gravedad" con un total de entrada de diez variables en una matriz de $1 \times 10$ de entrada, de $1 \times 1$ de salida y 36 reglas. Con este método se tiene en cuenta información relacionada con la función de pertenencia $\mu \mathrm{B}$. Se toma la media de todos los pesos, que es el valor medio del conjunto.

\section{Recopilación y análisis de datos}

Para evaluar bien y entender mejor el seguimiento de la recopilación de datos, nos hemos apoyado también en algunos sitios especializados para segmentar y analizar los componentes de sitios web de las empresas siderúrgicas chinas, tales como Page Rank http://checkpagerank.net, Internet Archive https://web.archive.org/web, Google Analytics https://www.google.com/analytics/web, Site Analysis http://uitest.com, la velocidad de la carga como el Pigdon http://tools.pingdom.com y el análisis de links del sitio web https://moz.com etc.

Desde la tabla 4, a cada métrica de evaluación se le asigna un grado de pertenencia entre 0 y 1 como se muestra también en la Tabla 5. La pertenencia es la principal característica contributiva a la incertidumbre y el rendimiento de los valores lingüísticos representa el desempeño de las $7 \mathrm{C}$ de los sitios web de las empresas siderúrgicas chinas. Esto significa el grado de rendimiento para el evaluador. A cada métrica de evaluación se le asigna un juicio cualitativo para determinar el grado de desempeño de los sitios web de las empresas siderúrgicas chinas en la categoría métrica seleccionada.

El rendimiento de los valores lingüísticos de los conjuntos difusos en este estudio están clasificados como muy pobre, inadecuado, mediano, bien y excelente (véase en la tabla 4). Estos juicios cualitativos se llaman valores lingüísticos y 
tienen el fin de construir protocolos de gestión o reglas de decisión, y se muestran a continuación:

Tabla 4. Valores lingüísticos utilizados para la evaluación

\begin{tabular}{|c|c|c|}
\hline $\begin{array}{c}\text { El grado de } \\
\text { pertenencia }\end{array}$ & $\begin{array}{c}\text { Término de } \\
\text { interpretación }\end{array}$ & Descripción de interpretaciones \\
\hline $0-0.19$ & Muy pobre & $\begin{array}{c}\text { La disponibilidad de los componentes de la interfaz de usuario es } \\
\text { muy pobre, con un promedio de menos de 2 componentes } \\
\text { disponibles }\end{array}$ \\
\hline $0.2-0.39$ & Inadecuado & $\begin{array}{c}\text { Componentes de interfaz de usuario son inadecuadamente } \\
\text { disponibles, con un promedio de menos de 4, pero más de } 2 \\
\text { componentes }\end{array}$ \\
\hline $0.4-0.59$ & Mediano & $\begin{array}{c}\text { Componentes de interfaz de usuario son moderadamente } \\
\text { disponibles, con un promedio de menos de 6, pero más de } 4 \\
\text { componentes. }\end{array}$ \\
\hline $0.6-0.79$ & Bien & $\begin{array}{c}\text { Componentes de interfaz de usuario son suficientemente disponibles, } \\
\text { con un promedio de menos de 8, pero más de 6 componentes. }\end{array}$ \\
\hline $0.8-1$ & Excelente & $\begin{array}{c}\text { Componentes de interfaz de usuario están disponibles a un nivel } \\
\text { muy alto, con un promedio de menos de diez, pero más que } 8 \\
\text { componentes. }\end{array}$ \\
\hline
\end{tabular}

Fuente: Elaboración propia.

Por tanto, los valores lingüísticos forman un conjunto difuso nuevo como $\mathrm{C}$, que toma valores en el universo con un intervalo de $[0,1]$, tal que: $\mathrm{f} \mathrm{C}(\mathrm{B})=\{0-$ $0.19 ; 0.2-0.39 ; 0.4-0.59 ; 0.6-0.79 ; 0.8-1$ \}

Con la finalidad de obtener medias para cada tratamiento, el siguiente paso es evaluar el desempeño de cada empresa siderúrgica por cada métrica de evaluación creada por el evaluador, como se muestra el desempeño de las $7 \mathrm{C}$ de sitios web de las 25 mejores empresas fabricantes del sector de acero de China en la tabla $5 \mathrm{y}$ el desempeño de las $7 \mathrm{C}$ de sitios web de las 25 mejores empresas intermediarias del dicho sector como se encuentra en la tabla 6 .

Según las reglas difusas que hemos descrito anteriormente, con el fin de describir el nivel de desempeño, hemos establecido una implicación de la relación difusa entre la métrica y el desempeño de sitios web de las empresas siderúrgicas chinas. Según esa implicación, se establece un valor mínimo de rendimiento asignado a todas las empresas seleccionadas en este estudio. La función de Max está aplicada para cada empresa, que es el conjunto de la evaluación métrica o la 
unión, también se toma la media de todos los pesos, que es el valor medio del conjunto como se muestra en la tabla 5 y 6 a continuación:

Tabla 5. La evaluación del desempeño de las 7C de sitios web de las 25 mejores empresas fabricantes del sector de acero de China

\begin{tabular}{|l|l|l|l|l|l|l|l|l|l|l|}
\hline & C 1 & C 2.1 & C 2.2 & C 2.3 & C 3 & C4 & C5 & C6.1 & C6.2 & C7 \\
\hline F 1. & 0.5 & 0.6 & 0.4 & 0.7 & 0 & 0.1 & 0.5 & 0.0019031 & 0.6 & 0 \\
\hline F 2. & 0.5 & 0.55 & 0.3 & 0.1 & 0 & 0.1 & 0.4 & 0.000648 & 0.4 & 0 \\
\hline F 3. & 0.7 & 0.65 & 0.5 & 0.4 & 0 & 0.1 & 0.5 & 0.141932 & 0.7 & 0.2 \\
\hline F 4. & 0.5 & 0.5 & 0.5 & 0.4 & 0 & 0.1 & 0.6 & 0.010961 & 0 & 0 \\
\hline F 5. & 0.6 & 0.6 & 0.3 & 0.3 & 0 & 0 & 0.4 & 0.035173 & 0.4 & 0 \\
\hline F 6. & 0.7 & 0.7 & 0.5 & 0.3 & 0 & 0 & 0.4 & 0.314649 & 0.4 & 0 \\
\hline F 7. & 0.7 & 0.7 & 0.5 & 0.4 & 0 & 0 & 0.4 & 0.035193 & 0 & 0.8 \\
\hline F 8. & 0.7 & 0.8 & 0.6 & 0.2 & 0 & 0.1 & 0.4 & 0.021626 & 0.5 & 0.9 \\
\hline F 9. & 0.6 & 0.7 & 0.5 & 0.4 & 0 & 0 & 0.4 & 0.266810 & 0.5 & 0.2 \\
\hline F 10. & 0.3 & 0.5 & 0.5 & 0.3 & 0 & 0 & 0.4 & 0.152356 & 0.5 & 0 \\
\hline F 11. & 0.5 & 0.6 & 0.5 & 0.2 & 0 & 0 & 0.4 & 0.000732 & 0 & 0 \\
\hline F 12. & 0.5 & 0.5 & 0.5 & 0.3 & 0 & 0 & 0 & 0.060802 & 0.5 & 0 \\
\hline F 13. & 0.7 & 0.6 & 0.5 & 0.3 & 0 & 0 & 0.4 & 0.070590 & 0.4 & 0 \\
\hline F 14. & 0.3 & 0.5 & 0.5 & 0.3 & 0 & 0 & 0.4 & 0.067888 & 0 & 0 \\
\hline F 15. & 0.6 & 0.7 & 0.5 & 0.3 & 0 & 0 & 0.4 & 0.008876 & 0.4 & 0 \\
\hline F 16. & 0.6 & 0.6 & 0.5 & 0.7 & 0 & 0 & 0.4 & 0.210149 & 0.5 & 0 \\
\hline F 17. & 0.6 & 0.7 & 0.5 & 0.3 & 0 & 0 & 0 & 0.039193 & 0 & 0 \\
\hline F 18. & 0.7 & 0.7 & 0.6 & 0.1 & 0 & 0.1 & 0.5 & 0.000094 & 0.3 & 0 \\
\hline F 19. & 0.7 & 0.7 & 0.5 & 0.2 & 0 & 0 & 0.4 & 0.059086 & 0.5 & 0.8 \\
\hline F 20. & 0.6 & 0.6 & 0.5 & 0.1 & 0 & 0 & 0.5 & 0.013817 & 0.3 & 0 \\
\hline F 21. & 0.4 & 0.7 & 0.5 & 0.4 & 0 & 0 & 0 & 0.000283 & 0.3 & 0 \\
\hline F 22. & 0.6 & 0.7 & 0.6 & 0.2 & 0 & 0 & 0.4 & 0.056848 & 0.4 & 0.2 \\
\hline F 23. & 0.3 & 0.6 & 0.5 & 0.3 & 0 & 0 & 0.4 & 0.290115 & 0.6 & 0.7 \\
\hline F 24. & 0.7 & 0.7 & 0.8 & 0.2 & 0 & 0.3 & 0.4 & 0.018103 & 0.4 & 0.8 \\
\hline F 25. & 0.6 & 0.6 & 0.6 & 0.2 & 0 & 0 & 0.4 & 0.013425 & 0.4 & 0 \\
\hline Fun & \\
\hline
\end{tabular}

Fuente: Elaboración propia. ${ }^{1}$

1 Nota: los datos para este análisis se recogieron en dos semanas (12/10/201526/10/2015) a través de las empresas seleccionadas de nuestra investigación. Puesto que 
Tabla 6. La evaluación del desempeño de $7 \mathrm{C}$ de sitios web de las 25 mejores empresas intermediarias del sector de acero de China

\begin{tabular}{|l|l|l|l|l|l|l|l|l|l|l|}
\hline & C 1 & C 2.1 & C 2.2 & $\begin{array}{l}\text { C } \\
2.3\end{array}$ & C3 & C4 & C5 & C6.1 & C6.2 & C7 \\
\hline M 1. & 0.8 & 0.8 & 0.7 & 0.6 & 0 & 0.5 & 0.7 & 0.136816 & 0.6 & 0 \\
\hline M 2. & 0.7 & 0.7 & 0.5 & 0.1 & 0 & 0 & 0.5 & 0.000006 & 0.4 & $0.5^{2}$ \\
\hline M 3. & 0.7 & 0.7 & 0.8 & 0.1 & 0 & 0.3 & 0.7 & 0.015814 & 0.5 & 0.7 \\
\hline M 4. & 0.8 & 0.7 & 0.7 & 0.5 & 0 & 0 & 0.7 & 0.001227 & 0.4 & 0 \\
\hline M 5. & 0.7 & 0.7 & 0.6 & 0.6 & 0 & 0 & $0^{3}$ & 0.171961 & 0.5 & 0 \\
\hline M 6. & 0.4 & 0.8 & 0.6 & 0.2 & 0 & 0 & 0.5 & 0.013206 & 0.4 & 0 \\
\hline M 7. & 0.7 & 0.7 & 0.6 & 0.2 & 0 & 0 & 0.5 & 0.026964 & 0.4 & 0 \\
\hline M 8. & 0.4 & 0.4 & 0.4 & 0.1 & 0 & 0 & 0.5 & 0 & 0 & 0 \\
\hline M 9. & 0.6 & 0.4 & 0.5 & 0.1 & 0 & 0 & 0.5 & 0.000876 & 0.3 & 0 \\
\hline M 10. & 0.3 & 0.4 & 0.4 & 0.1 & 0 & 0 & 0.5 & 0.000001 & 0 & 0 \\
\hline M 11. & 0.2 & 0.3 & 0.3 & 0.1 & 0 & 0 & 0.5 & 0 & 0 & 0 \\
\hline
\end{tabular}

cuyos sitios web se actualizan de forma continua, el seguimiento de los estudios que utilizan estos sitios podrían encontrar algunas diferencias paródicamente. Aunque estas empresas representan las empresas más grandes del país, pero sus prácticas de sitios web no son necesariamente representativos de los sitios web de las organizaciones en general del dicho país. También hay que señalar que los resultados de C6 (la disponibilidad de enlaces externos a otros sitios), obtenido desde PageRank, que está disponible en http://checkpagerank.net. En este estudio, suponemos que el número más máximo de páginas externas que pueda llegar un sitio web de una empresa mundial como el caso de nosotros es cien mil, y los datos estadísticos de este apartado son dividido por $10^{6}$ para ajustar entre los valores numéricos 0 y 1 . Se da un cero a los links y páginas que no se pueden abrir, estén bloqueados y no llevan la información indicada correctamente. Por ejemplo: en el caso de F12, que no tiene un apartado donde explica el número de contacto o correo de la empresa, por eso su C 5 es un cero; F17 no dispone el teléfono, ni el correo de la empresa en el apartado del contacto, por eso cuyo C 5 también es un cero). Otra cosa más importante para este análisis es el caso de C7, que sólo se observaron los sitio web elegidos si tiene un link que nos dirige hacia sus transacciones bancarias que realizan en línea o no, ya que como el caso de Baosteel tiene su plataforma de Comercio, pero en su sitio web de la empresa no se encuentra un link que nos orienta hacia su plataforma electrónica de comercio.

${ }^{2}$ Nota: C7 de M2 tiene un apartado que menciona cuya plataforma de comercio electrónico de la empresa, pero no se encuentra un link activo, por eso cuya nota es 0.5.

${ }^{3}$ Nota: En el apartado de C4 de M5, sólo se encuentra un télefono abajo de la pantalla. 


\begin{tabular}{|c|c|c|c|c|c|c|c|c|c|c|}
\hline M 12. & 0.7 & 0.5 & 0.4 & 0.2 & 0 & 0 & 0.5 & 0.000179 & 0.3 & 0 \\
\hline M 13. & 0.7 & 0.6 & 0.6 & 0.3 & 0 & 0 & 0.5 & 0.002420 & 0.3 & 0 \\
\hline M 14. & 0.2 & 0.2 & 0.3 & 0.1 & 0 & 0 & 0.1 & 0 & 0 & 0 \\
\hline M 15. & 0.7 & 0.6 & 0.6 & 0.2 & 0 & 0 & 0.7 & 0.026428 & 0.3 & 0 \\
\hline M 16. & 0.2 & 0.1 & 0.1 & 0 & 0 & 0 & 0.3 & 0 & 0 & 0 \\
\hline M 17. & 0.5 & 0.6 & 0.5 & 0.2 & 0 & 0 & 0.6 & 0 & 0 & 0 \\
\hline M 18. & 0.5 & 0.5 & 0.4 & 0.1 & 0 & 0 & 0.7 & 0.013760 & 0.4 & 0 \\
\hline M 19. & 0.2 & 0.25 & 0.2 & 0.1 & 0 & 0 & 0.5 & 0 & 0 & 0 \\
\hline M 20. & 0.6 & 0.7 & 0.6 & 0.1 & 0 & 0 & 0.5 & 0.015525 & 0.2 & 0 \\
\hline M 21. & 0.3 & 0.4 & 0.4 & 0.1 & 0 & 0 & 0.6 & 0 & 0 & 0 \\
\hline M 22. & 0.7 & 0.7 & 0.6 & 0.1 & 0 & 0 & 0.5 & 0.000163 & 0.2 & 0 \\
\hline M 23. & 0.75 & 0.7 & 0.6 & 0.1 & 0 & 0 & 0.5 & 0.001152 & 0.4 & 0 \\
\hline M 24. & 0.5 & 0.71 & 0.6 & 0.2 & 0 & 0 & 0.5 & 0.070983 & 0.3 & 0.5 \\
\hline M 25. & 0.48 & 0.4 & 0.5 & 0.2 & 0 & 0 & 0.5 & 0 & 0 & 0 \\
\hline
\end{tabular}

Fuente: Elaboración propia ${ }^{4}$.

\section{Resultados y discusión}

El modelo de lógica difusa presenta y proporciona una forma flexible de evaluación del rendimiento de sitio web y también permite la toma de decisiones mediante una amplia gama de variables lingüísticas. En esta parte del trabajo, utilizamos tres conjuntos difusos de entrada (unión, intersección y promedio), que son 10 variables de entrada (10 medidas seleccionadas del 7C), para inferir el tipo de conjunto difuso de salida para cada empresa seleccionada, que es el rendimiento de diseño de sitio web de cada empresa tanto fabricante como intermediaria.

4 Nota: los datos para este análisis se recogieron en dos semanas (12/10/201526/10/2015) a través de las empresas seleccionadas de nuestra investigación. Puesto que cuyos sitios web se actualizan de forma continua, el seguimiento de los estudios que utilizan estos sitios podrían encontrar algunas diferencias paródicamente. Aunque estas empresas representan las empresas intermediarias más grandes del país, pero sus prácticas de sitios web no son necesariamente representativos de los sitios web de las organizaciones en general del dicho país. Se da un cero a los links y páginas que no se pueden abrir, o estén bloqueados, también para sitios que no muestran la información indicada correctamente. 
Las tablas 7, 8, 9 y 10 están basadas en las reglas creadas en la fuzyficación de nuestro estudio y muestran el resumen del desempeño de las $7 \mathrm{C}$ del diseño de sitios web de las empresas tanto fabricantes como intermediarias del sector de acero de China. En la tabla 11 se refleja una comparación de las variables de la entrada del rendimiento de las $7 \mathrm{C}$ de sitios web de las 25 empresas fabricantes y las 25 empresas intermediarias del dicho sector.

Tabla 7. El resumen de las variables de entrada del rendimiento del sitio web de las empresas fabricantes del sector de acero de China

\begin{tabular}{|l|l|l|l|}
\hline Nombre de la empresa & Unión & Intersección & Promedio \\
\hline F1. Shanghai Baosteel Group Corporation & 0.8 & 0 & 0.34 \\
\hline F2. Hebei Iron \& Steel Group & 0.55 & 0 & 0.24 \\
\hline F3. Shagang Group & 0.7 & 0.1 & 0.38 \\
\hline F4. Wuhan Iron and Steel Group & 0.6 & 0 & 0.26 \\
\hline F5. Bohai steel group & 0.6 & 0 & 0.26 \\
\hline F6. Shougang group & 0.7 & 0 & 0.26 \\
\hline F7. Xinxing Cathay International Group & 0.8 & 0 & 0.35 \\
\hline F8. Ansteel group & 0.9 & 0.1 & 0.42 \\
\hline F9. Taiyuan Iron \& Steel Group & 0.7 & 0 & 0.36 \\
\hline F10. Jiuquan Iron \& Steel Group & 0.5 & 0 & 0.27 \\
\hline F11. Shandong Iron \& Steel Group & 0.6 & 0 & 0.22 \\
\hline F12. Benxi Iron \& Steel Group & 0.5 & 0 & 0.24 \\
\hline F13. Zenith Steel Group & 0.7 & 0 & 0.3 \\
\hline F14. Hangzhou Iron \& Steel Group & 0.5 & 0 & 0.2 \\
\hline F15. Valin group & 0.7 & 0 & 0.3 \\
\hline F16. Magang Group & 0.7 & 0 & 0.35 \\
\hline F17. Jianlong Group & 0.7 & 0 & 0.2 \\
\hline F18. Sino Giant Group & 0.7 & 0 & 0.3 \\
\hline F19. Nanjing Iron \& Steel Group & 0.8 & 0 & 0.39 \\
\hline F20. Jiangsu Xicheng Sanlian Holding Group & 0.6 & 0 & 0.26 \\
\hline F21. Fosun Group & 0.7 & 0 & 0.23 \\
\hline F22. Jinxi Group & 0.7 & 0 & 0.31 \\
\hline F23. Baogang Group & 0.7 & 0 & 0.37 \\
\hline F24. Hebei Jingye Group & 0.8 & 0 & 0.33 \\
\hline F25. Tranvic Group & 0.6 & 0 & \\
\hline & & 0 & \\
\hline
\end{tabular}

Fuente: Elaboración propia. 
Tabla 8. El resumen del rendimiento del sitio web de las empresas fabricantes del sector de acero de China a través del clasificador Fuzzy

\begin{tabular}{|l|l|l|l|l|}
\hline & $\begin{array}{l}\text { Función } \\
\text { pertenencia: da } \\
\text { unión }\end{array}$ & $\begin{array}{l}\text { Función de pertenencia: } \\
\text { la intersección }\end{array}$ & $\begin{array}{l}\text { Promedio de los } \\
\text { resultados }\end{array}$ & $\begin{array}{l}\text { Clasificación } \\
\text { rendimiento de Fuzzy }\end{array}$ \\
\hline F 1. & Excelente & Muy pobre & Inadecuado & Inadecuado \\
\hline F 2. & Mediano & Muy pobre & Inadecuado & Inadecuado \\
\hline F 3. & Bien & Muy pobre & Inadecuado & Inadecuado \\
\hline F 4. & Bien & Muy pobre & Inadecuado & Inadecuado \\
\hline F 5. & Bien & Muy pobre & Inadecuado & Inadecuado \\
\hline F 6. & Bien & Muy pobre & Inadecuado & Inadecuado \\
\hline F 7. & Excelente & Muy pobre & Inadecuado & Inadecuado \\
\hline F 8. & Excelente & Muy pobre & Mediano & Mediano \\
\hline F 9. & Bien & Muy pobre & Inadecuado & Inadecuado \\
\hline F 10. & Mediano & Muy pobre & Inadecuado & Inadecuado \\
\hline F 11. & Mediano & Muy pobre & Inadecuado & Inadecuado \\
\hline F 12. & Mediano & Muy pobre & Inadecuado & Inadecuado \\
\hline F 13. & Bien & Muy pobre & Inadecuado & Inadecuado \\
\hline F 14. & Mediano & Muy pobre & Inadecuado & Inadecuado \\
\hline F 15. & Bien & Muy pobre & Inadecuado & Inadecuado \\
\hline F 16. & Bien & Muy pobre & Inadecuado & Inadecuado \\
\hline F 17. & Bien & Muy pobre & Inadecuado & Inadecuado \\
\hline F 18. & Bien & Muy pobre & Inadecuado & Inadecuado \\
\hline F 19. & Excelente & Muy pobre & Inadecuado & Inadecuado \\
\hline F 20. & Bien & Muy pobre & Inadecuado & Inadecuado \\
\hline F 21. & Bien & Muy pobre & Inadecuado & Inadecuado \\
\hline F 22. & Bien & Muy pobre & Mediano & Inadecuado \\
\hline F 23. & Bien & Muy pobre & Inadecuado \\
\hline F 24. & Excelente & Muy pobre & Mediano \\
\hline F 25. & Bien & & Inadecuado \\
\hline
\end{tabular}

Fuente: Elaboración propia. 
Tabla 9. El resumen de las variables de entra del rendimiento del sitio web de las empresas intermediarias del sector de acero de China

\begin{tabular}{|c|c|c|c|}
\hline & Unión & Intersección & Promedio \\
\hline M 1. China Railway Materials Company Limited & 0.8 & 0 & 0.49 \\
\hline M 2. Minmetals Development Co., Limited & 0.7 & 0 & 0.34 \\
\hline M 3.Zhejiang Materials Industry Group & 0.8 & 0 & 0.45 \\
\hline M 4. Hangzhou Ciec Group & 0.8 & 0 & 0.38 \\
\hline M 5. Sinosteel Corporation & 0.7 & 0 & 0.33 \\
\hline M 6.Huanan Group & 0.8 & 0 & 0.29 \\
\hline M 7.Xiangyu Group & 0.7 & 0 & 0.31 \\
\hline M 8. Southern Building Materials Co., Ltd. & 0.5 & 0 & 0.18 \\
\hline M 9.Junhe Group & 0.6 & 0 & 0.24 \\
\hline M 10. $\quad$ Shanghai Miaoshi Group Co.,Ltd & 0.5 & 0 & 0.17 \\
\hline M 11. Shanghai Baiying Iron \& Steel Group & 0.5 & 0 & 0.14 \\
\hline M 12. Xiamen C\&D Material Co., Ltd & 0.7 & 0 & 0.26 \\
\hline M 13. $\quad$ Huaye Iron \& Steel Group & 0.7 & 0 & 0.3 \\
\hline M 14. $\quad$ Shanghai Minlurun Trade Co.,Ltd & 0.3 & 0 & 0.09 \\
\hline M 15. $\quad$ Chang Jiang Steel Pipe Co., Ltd & 0.7 & 0 & 0.31 \\
\hline M 16. $\quad$ Shanghai Ningjin Iron \& Steel Co., Ltd & 0.3 & 0 & 0.07 \\
\hline M 17. Shanghai Jushen Metal Industry Co., Ltd & 0.6 & 0 & 0.24 \\
\hline M 18. $\quad$ Shanghai Shunye Steel Co., Ltd & 0.7 & 0 & 0.26 \\
\hline M 19. Shanghai Ronghui Wuzi Co., Ltd & 0.5 & 0 & 0.13 \\
\hline M 20. $\quad$ Golden Jumping Group & 0.7 & 0 & 0.27 \\
\hline M 21. Shanghai Qiangguan Steel Co., Ltd & 0.6 & 0 & 0.2 \\
\hline M 22. $\quad$ Qixin Group of Hebei & 0.7 & 0 & 0.28 \\
\hline M 23. $\quad$ Zhanzhi Group & 0.75 & 0 & 0.3 \\
\hline M 24. $\quad$ Beijing Zhongtie Construct Materials Ltd & 0.71 & 0 & 0.33 \\
\hline M 25. Shanghai Mingyao International Co., Ltd & 0.5 & 0 & 0.17 \\
\hline
\end{tabular}

Fuente: Elaboración propia. 
Tabla 10. El resumen del rendimiento del sitio web de las empresas intermediarias del sector de acero de China a través de Fuzzy

\begin{tabular}{|c|c|c|c|c|}
\hline & $\begin{array}{lr}\text { Función } & \mathrm{de} \\
\text { pertenencia: } & \text { la } \\
\text { unión } & \\
\end{array}$ & $\begin{array}{l}\text { Función de pertenencia: } \\
\text { la intersección }\end{array}$ & $\begin{array}{l}\text { Promedio de los } \\
\text { resultados }\end{array}$ & $\begin{array}{l}\text { Clasificación de } \\
\text { rendimiento de Fuzzy }\end{array}$ \\
\hline M 1. & Excelente & Muy pobre & Mediano & Mediano \\
\hline M 2. & Bien & Muy pobre & Inadecuado & Inadecuado \\
\hline M 3. & Excelente & Muy pobre & Mediano & Mediano \\
\hline M 4. & Excelente & Muy pobre & Inadecuado & Inadecuado \\
\hline M 5. & Bien & Muy pobre & Inadecuado & Inadecuado \\
\hline M 6. & Excelente & Muy pobre & Inadecuado & Inadecuado \\
\hline M 7. & Bien & Muy pobre & Inadecuado & Inadecuado \\
\hline M 8. & Mediano & Muy pobre & Muy pobre & Muy pobre \\
\hline M 9. & Bien & Muy pobre & Inadecuado & Inadecuado \\
\hline M 10. & Mediano & Muy pobre & Muy pobre & Muy pobre \\
\hline M 11. & Mediano & Muy pobre & Muy pobre & Muy pobre \\
\hline M 12. & Bien & Muy pobre & Inadecuado & Inadecuado \\
\hline M 13. & Bien & Muy pobre & Inadecuado & Inadecuado \\
\hline M 14. & Inadecuado & Muy pobre & Muy pobre & Muy pobre \\
\hline M 15. & Bien & Muy pobre & Inadecuado & Inadecuado \\
\hline M 16. & Inadecuado & Muy pobre & Muy pobre & Muy pobre \\
\hline M 17. & Bien & Muy pobre & Inadecuado & Inadecuado \\
\hline M 18. & Bien & Muy pobre & Inadecuado & Inadecuado \\
\hline M 19. & Mediano & Muy pobre & Muy pobre & Muy pobre \\
\hline M 20. & Bien & Muy pobre & Inadecuado & Inadecuado \\
\hline M 21. & Bien & Muy pobre & Inadecuado & Inadecuado \\
\hline M 22. & Bien & Muy pobre & Inadecuado & Inadecuado \\
\hline M 23. & Bien & Muy pobre & Inadecuado & Inadecuado \\
\hline M 24. & Bien & Muy pobre & Inadecuado & Inadecuado \\
\hline M 25. & Mediano & Muy pobre & Muy pobre & Muy pobre \\
\hline
\end{tabular}

Fuente: Elaboración propia. 
Tabla 11. Comparación de los resultados de rendimiento de $7 \mathrm{C}$ de sitios web entre las empresas fabricantes y intermediarias del sector de acero de China

\begin{tabular}{|l|l|l|}
\hline Término & Empresas fabricantes & Empresas intermediarias \\
\hline MP (Muy pobre) & 0 & 7 \\
\hline IA (Inadecuado) & 23 & 16 \\
\hline ME (Mediano) & 2 & 2 \\
\hline BE (Bien) & 0 & 0 \\
\hline EX (Excelente) & 0 & 0 \\
\hline
\end{tabular}

Fuente: Elaboración propia.

El mantenimiento de un sitio web con fines de comercio electrónico parece ser una opción popular para las empresas industriales. Sin embargo, vemos solamente la presencia de algunas de las 10 variables creadas en 50 empresas siderúrgicas chinas evaluadas. Un factor visible en casi todos los sitios web evaluados fue la ausencia de los dos subcomponentes importantes como la comunidad y la personalización. Es evidente que ningún sitio web esté interesado en mantener una comunicación interactiva tanto de usuario a usuario como del sitio web al usuario.

Ningún sitio web de las empresas elegidas en esta investigación tiene la disponibilidad de mantener una comunicación absoluta y viva con sus usuarios, excepto de un tablero de opiniones, porque ninguna de estas empresas tiene un sitio web dinámico, todos son estáticos y no tienen la capacidad de ajustarse. Dos de las empresas elegidas, ni siquiera tienen un domino propio, sino que alquila el sitio de otras empresas.

La conversión también ha sido considerada como una tarea técnicamente muy compleja por los diseñadores de las empresas elegidas, ya que dos componentes para la evaluación de la conversión en este estudio, son la adaptación (la capacidad de los sitios web de adaptar en sí) y personalización (la capacidad de los sitios que se adapta por cada usuario). No se refleja la adaptación, ni la personalización en todos los sitios examinados, porque son sitios web estáticos y no dinámicos.

En la parte del rango de las páginas, ninguna de las empresas seleccionadas tienen una pertenencia muy alta, que llegue al "1", dentro de las cuales se encuentra Shagang Group, Baosteel y Hebei Iron \& Steel Group, y están calificadas como medio alto de calidad de página por Google, que son las que tienen mejor rendimiento en esta evaluación, comparando el resto de las empresas participantes. El sitio web de la empresa intermediaria "China Railway Materials Company" también está calificada por Google como nivel de calidad medio-alto. 
Otro factor importante fue el componente de Comercio, donde se evaluó la capacidad de las transacciones bancarias que realizan en línea, ya que muy pocas empresas del sector de acero de China están capacitadas en este aspecto, sólo 6 empresas fabricantes y una intermediaria tienen la posibilidad de realizar transacciones bancarias en línea, y el resto de las empresas evaluadas ni siquiera tienen un link activo hacia una plataforma de comercio electrónico, que les facilite ventas en línea.

Las razones de esta baja cifra también pueden venir motivadas por las debilidades en la infraestructura de información, por razones de seguridad, por las bajas tasas de consumo de tecnología de la información, así como por las bajas tasas de penetración de Internet en el sector de acero. También un motivo adicional es la inversión sustancial que implica en el mantenimiento de un sitio web de comercio electrónico.

Con esta asignación de resultados, se puede ver que ninguna de las empresas siderúrgicas tiene un tratamiento "excelente" o "bien" de 7C de diseño de sitio web, sin distinguir entre empresa fabricante o intermediaria. Comparando el rendimiento de diseño del sitio web entre las empresas fabricantes y intermediarias, los fabricantes tienen un mejor rendimiento que los intermediarios, ya que 7 empresas intermediarias tienen un tratamiento "muy pobre" de 7C, mientras que ninguna de las fabricantes está calificadas como "muy pobre". Hay que destacar que de las 50 empresas siderúrgicas evaluadas en este estudio, sólo 4 empresas (2 fabricantes y 2 intermediarios) tienen un rendimiento "mediano", que es la máxima punto en esta evaluación. En general, en la mayoría de los casos el rendimiento en cuanto a diseño de $7 \mathrm{C}$ de sitio web de las empresas siderúrgicas chinas está considerado como "inadecuado".

\section{CONCLUSIONES}

Según los resultados obtenidos, se puede ver que analizar los sitio web de las empresas siderúrgicas chinas mediante la lógica difusa es útil en la explicación tanto de fenómenos de enunciados teóricos como de enunciados empíricos. Como se ha señalado, el modelo de las 7C de Jeffrey Rayport y Bernard Jaworski (2001) es eficaz para medir el rendimiento del diseño del sitio web, que se reflejan las dimensiones de la experiencia y determinan el potencial de sitio web para la captación del interés del cliente.

Los resultados aportados hasta ahora justifican, por medio de la lógica difusa, que todas las empresas elegidas en nuestro estudio tienen un sitio web de tipo estático, no tienen la posibilidad de realizar transacciones bancarias en línea, y algunas empresas evaluadas ni siquiera tienen un link activo que dirija a una plataforma de comercio electrónico, que le facilite ventas en línea. No están 
dispuestas a mantener una comunicación interactiva, ni tienen la capacidad de adaptación y de personalización.

Por medio de la lógica difusa, se vuelve a comprobar, al igual que en el capítulo anterior, que las bajas tasas de penetración de comercio electrónico en el sector de acero de China pueden ser las debilidades en la infraestructura de información, razones de seguridad, y las bajas tasas de consumo de tecnología de la información. También puede ser la gran inversión sustancial implicados en el mantenimiento de un sitio web de comercio electrónico.

En conclusión, los resultados de la evaluación fueron muy alentadores, porque la lógica difusa es una herramienta eficaz para la toma de decisiones cuando se encuentra con rangos de datos que en ocasiones son de difícil interpretación por la lógica normal. En el caso de sitio web de las empresas siderúrgicas chinas donde intervienen varios factores (entre ellos las 10 medidas seleccionadas de 7C), la lógica difusa se convierte en una excelente alternativa para la evaluación del rendimiento del diseño del sitio web de las empresas elegidas, pues trabaja con datos lingüísticos imprecisos y los transforma en resultados medibles que brindan respuestas a determinados niveles de rendimiento de diseño de sitio web de las empresas siderúrgicas chinas, como es el caso de esta investigación.

\section{BIBLIOGRAFÍA}

ALARCÓN, M. \& LORENZO, C. (2012) "Diferencias entre usuarios y no usuarios de redes sociales virtuales en la Web 2.0" en Enl@ce: Revista Venezolana de Información, Tecnología y Conocimiento, 9 (2)., pp. 31-49.

ESTRADE, N. et al. (2013) Marketing digital: Marketing móvil, SEO y analítica web. Madrid: Grupo Anaya, S.A., pp. 22-150.

FOUCHARD, G. (2002) Cómo vender más en su Web. Barcelona: Ediciones Gestión 2000, S.A., pp. 20-88.

GÓMEZ, B. \& TAPIA, A. (2011) Análisis de la comunicación de páginas Web en las cincuenta mayores empresas del sector publicitario español. Madrid: MHCJ n 2., pp. 40-180.

JANG, J. S. R., SUN C. T., \& MIZUTANI E. (1997) Nuero-Fuzzy and soft computing. A computational approach to learning and machine intelligence. London, UK: Prentice-Hall., pp.10-99

KYUN, O. Y. (2013) Estudio de la gestión del proceso turístico ante un entorno multicultural mediante Lógica Borrosa (Fuzzy Logic). Tesis doctoral. Barcelona, Departamento de Economía y Organización de Empresas, Facultad de Economía y Empresas, Universitat de Barcelona., pp. 27-254.

LEÓN DE MORA, C. (2009) Método general de Extracción de Información basado en el uso de Lógica Borrosa. Aplicación en portales web. Tesis 
doctoral. Sevilla, Departamento de Tecnología Electrónica, Universidad de Sevilla., pp. 63-76.

NIETO, B. G. \& TAPIA, F. (2011) “Análisis de la comunicación de páginas Web en las cincuenta mayores empresas del sector publicitario español " [pdf] Miguel Hernández Comunication Journal., pp. 119-140.

PRAT, M. (2013) SEO - Posicionamiento de su sitio Web en Google y otros buscadores. 3ra Edición. Barcelona: Ediciones ENI., pp. 25-150.

RANAWAKA, R. A. K. (2008) "Comparing the User-Interface Design of Banking Websites; A Survey on the Sri Lankan Banking Sector", 4th International Conference on Information and Automation for Sustainability. PP: 157-162. Available from: LSE Portal: LSE Digital Library. [Accessed 23 June 2015].

RAYPORT, J. F. \& JAWORSKI, B. J. (2001) e-Commerce. México: McGrawHill., pp.133-180.

ROPERO, R. J. (2009) Método general de Extracción de Información basado en el uso de Lógica Borrosa. Aplicación en portales web. Sevilla, Departamento de Tecnología Electrónica, Escuela Técnica Superior de Ingeniería Informática, Universidad de Sevilla., pp. 67-156.

STERNE, J. (1998) La publicidad en Web. Edo. de México: Prentice-Hall Hispanoamericana, S.A., pp. 352-369.

VERA, M. L. (2014) Aplicación y comparación de metodologías multicriterio (AHP y Fuzzy Logic) en la selección de tecnología postcosecha para pequeños productores de cacao. Valencia, Departamento de Economía y Ciencias Sociales, Universitat Politècnica de Valencia., pp. 97-125.

ZADEH, L. A. (1965) Fuzzy Sets. Information and Control [Online] Available at: $<$ http://www.cs.berkeley.edu/ zadeh/papers/Fuzzy\%20SetsInformation\%20and\%20Control-1965.pdf $>$ [ Accessed 20 July 2015].

ZADEH, L. A. (1975): The concept of a linguistic variable and its application to approximate reasoning, Information science, Part I, Vol. 8, pp.199-249, Part II, Vol.8, pp.301-357, Part III, Vol. 9, pp. 20-340. 